\title{
Spotlight on naldemedine in the treatment of opioid-induced constipation in adult patients with chronic noncancer pain: design, development, and place in therapy
}

This article was published in the following Dove Press journal: Journal of Pain Research

\author{
Emily K Stern \\ Darren M Brenner \\ Division of Gastroenterology, \\ Department of Internal Medicine, \\ Northwestern University Feinberg \\ School of Medicine, Chicago, IL, USA
}

Correspondence: Darren M Brenner Division of Gastroenterology, Department of Internal Medicine, Northwestern University Feinberg School of Medicine, 676 North Saint Clair Avenue, Suite 1400, Chicago, IL 606II, USA

Tel + I 3126955620

Fax + I 3126957095

Email darren-brenner@northwestern.edu

\begin{abstract}
Opioid-induced constipation (OIC) is an increasingly prevalent problem in the USA due to the growing use of opioids. A novel class of therapeutics, the peripherally acting $\mu$-opioid receptor antagonists (PAMORAs), has been developed to mitigate the deleterious effects of opioids in the gastrointestinal tract while maintaining central analgesia and minimizing opioid withdrawal. This review aimed to summarize the literature on naldemedine, the third PAMORA to gain US Food and Drug Administration (FDA) approval for the treatment of OIC in adults with chronic noncancer pain-related syndromes. Naldemedine has a chemical structure similar to naltrexone, an opioid receptor antagonist, with chemical modifications that limit its ability to cross the blood-brain barrier. Naldemedine was evaluated in two Phase II and three Phase III clinical trials prior to gaining FDA approval. In two pivotal identical Phase III trials, COMPOSEI (NCT 01965158) and COMPOSE-II (NCT 01993940), patients receiving naldemedine were significantly more likely to respond when compared with placebo (COMPOSE-I: 47.6 vs $34.6 \%$, $P=0.002$ and COMPOSE-II: 52.5 vs $33.6 \%, P<0.0001)$. The most frequent adverse events were abdominal pain ( $8 \%$ ) and diarrhea $(7 \%)$ - rates similar to the other PAMORAs. Based on the available data, naldemedine appears to be an effective and safe drug for the treatment of OIC in adults with chronic noncancer pain.
\end{abstract}

Keywords: opioid-induced constipation, constipation, opioids, opioid antagonist, naldemedine, peripherally acting u-opioid receptor antagonists, PAMORA

\section{Introduction}

Chronic pain is a common and growing problem. In 2012, 25.3 million adults living in the USA ( $11.2 \%$ of the population) reported chronic daily pain ${ }^{1}$ and more recent analyses suggest that opioid use for chronic noncancer pain has become increasingly prevalent. ${ }^{2}$ Unfortunately, while these analgesics are effective for treating pain, their side effects can be serious and limit tolerability. Constipation, the most common side effect due to chronic opioid use, occurs in $40-80 \%$ of patients utilizing these agents. ${ }^{3}$ A new class of therapeutics, the peripherally acting $\mu$-opioid receptor antagonists (PAMORAs), has been developed to directly counteract this deleterious effect. This review focuses on naldemedine, the most recent PAMORA to be approved by the US Food and Drug Administration (FDA) for the treatment of opioid-induced constipation (OIC) in adult patients with chronic noncancer pain. 


\section{OIC}

A working definition for OIC was recently proposed by a multispecialty consensus and solidified in the Rome IV guidelines. ${ }^{4,5}$ According to Rome IV, OIC is defined as "new or worsening symptoms of constipation when initiating, changing, or increasing opioid therapy". To meet this definition, patients must experience at least two of the six symptoms used to define functional constipation (Table 1) and should rarely have loose stools without the use of laxatives. ${ }^{5}$ Recently, there has been an effort within the field to distinguish OIC from opioid-exacerbated constipation (OEC). In OIC, the complaint of constipation is isolated to opioid initiation, whereas in OEC, underlying constipation symptoms are worsened by opioid usage. ${ }^{3}$ In this review, the term OIC is used to describe a change in bowel patterns associated with opioid usage.

There is a high prevalence of OIC among patients with chronic noncancer pain. In a systematic review of placebo (PBO)-controlled trials involving patients with chronic noncancer pain on opioids, $\sim 41 \%$ of patients reported constipation as an adverse event (AE). ${ }^{6}$ Similarly, a populationbased survey of $>1,000$ patients revealed that $57 \%$ of these individuals reported constipation associated with the therapy. ${ }^{7}$ OIC may have a significant impact on the quality of life for patients on chronic opioids. ${ }^{4}$

The pathophysiology of OIC relates to the effect of opioids on receptors found in the gastrointestinal (GI) tract. Opioids induce analgesia predominately via their action on central opioid receptors, particularly the $\mu$-opioid receptors. ${ }^{3,4}$ These same $\mu$-opioid receptors line the gut mucosa and are found in the myenteric and submucosal plexus of the GI tract. ${ }^{8}$ The binding of exogenous opioids to the gut myenteric plexus decreases peristaltic contractions and slows GI transit. ${ }^{8}$ Activation of the submucosal plexus leads to increased water absorption and decreased fluid and electrolyte secretion. ${ }^{8}$ Both contribute to constipation. In addition, opioids increase anal sphincter tone and impair rectal relaxation with distension, which can cause issues with pelvic floor coordination during defecation. ${ }^{8}$
Traditional laxatives are considered the first-line therapy for OIC given their safety, low cost, and over-the-counter availability. ${ }^{4,5}$ However, there are no randomized controlled trials looking at laxative use in OIC. Furthermore, there is strong evidence that traditional laxatives are ineffective in patients on opioids. In a study of 322 patients taking daily opioids and laxatives, $81 \%$ reported constipation and 58\% reported straining with bowel movements despite laxative use. ${ }^{9}$ Given that many patients do not respond to laxatives, there has been motivation to develop additional therapeutic options for OIC patients. In 2013, the first and only non-PAMORA, lubiprostone, was approved by the FDA for this condition. ${ }^{10}$ Lubiprostone, a type- 2 chloride channel activator, indirectly functions by increasing luminal water and electrolyte secretion as well as gut motility, thus potentially counteracting some of the opioid side effects. ${ }^{11}$

Since then, the PAMORA class has been developed to directly target opioid receptors in the GI tract, potentially reversing the side effects of exogenous opioids without compromising central analgesia. The first two PAMORAs, FDA approved for the treatment of OIC in chronic noncancerrelated pain patients, were methylnaltrexone bromide (MNT) and naloxegol. MNT was initially FDA approved in subcutaneous (SC) form in 2008 for the treatment of OIC in patients with advanced illnesses receiving palliative care when standard laxatives failed and subsequently in SC and oral form in 2014 and 2016, respectively, for the treatment of OIC in patients using opioids for noncancer pain conditions. ${ }^{3}$ Naloxegol, a PEGylated derivative of the $\mu$-opioid antagonist naloxone, ${ }^{12}$ was FDA approved for OIC in patients with chronic noncancer pain in 2014., ${ }^{3,13}$ Naldemedine, the third in-class agent, gained approval in March 2017. ${ }^{14}$

\section{Naldemedine Design}

Naldemedine has a chemical structure similar to that of the opioid receptor antagonist naltrexone..$^{15}$ It consists of the naltrexone molecule with an added side chain, which increases its weight and polar surface area, thus limiting

Table I Rome IV diagnostic criteria for opioid-induced constipation

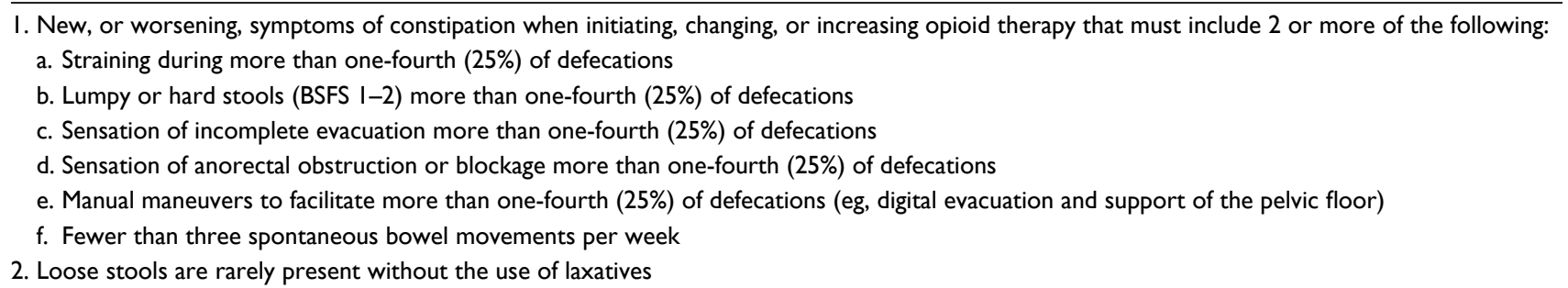

Notes: Patients must report new, or worsening, symptoms of constipation when initiating, changing, or increasing opioid therapy that must include at least two of the listed symptoms. In addition, patients must rarely have loose stools without the use of laxatives. Reprinted from Gastroenterology, 150(6), Lacy BE, Mearin F, Chang L, et al. Bowel disorders, 1393-1407.el 395, Copyright (2016), with permission from Elsevier. ${ }^{5}$

Abbreviation: BSFS, Bristol Stool Form Scale. 
its ability to cross the blood-brain barrier. ${ }^{16}$ Naldemedine is also a substrate of the P-glycoprotein efflux transporter, which limits its ability to concentrate in the central nervous system by actively transporting any penetrating naldemedine out of the central nervous system. ${ }^{16}$ Initial pharmacodynamic studies performed in rat models of OIC showed that naldemedine decreased intestinal transit time without effecting analgesia or inducing withdrawal. ${ }^{17,18}$ Pharmacokinetic studies in healthy and OIC patients revealed that naldemedine exposure increased in a dose-dependent manner, with steadystate levels reached within 2 days of administration. ${ }^{16}$ The drug is almost universally bound to albumin (94\%) and not removed by dialysis. ${ }^{16}$ Naldemedine is metabolized primarily by CYP3A4 to form nor-naldemedine, ${ }^{16}$ and in initial studies, administration of naldemedine with rifampin decreased naldemedine concentrations while administration with fluconazole increased them. ${ }^{17}$ Therefore, co-administration of naldemedine with CYP3A4 inducers should be avoided and co-administration of naldemedine with CYP3A4 inhibitors should be done only with careful monitoring. ${ }^{17}$ Neither naldemedine nor nor-naldemedine inhibits or induces the major CYP450 enzymes. ${ }^{16}$

\section{Development}

Five Phase II/III clinical trials were completed leading to FDA approval of naldemedine for use in OIC patients with chronic noncancer pain. The first Phase IIa (NCT 01122030) dose-escalation study was designed primarily for safety analysis. Seventy-two patients received a one-time dose of naldemedine $0.01,0.03,0.1,0.3,1.0$, or $3.0 \mathrm{mg}$ or PBO (nine patients per naldemedine cohort, 18 patients $\mathrm{PBO}) .{ }^{19}$ Treatment-emergent AEs (TEAEs) occurred more frequently in individuals receiving naldemedine than PBO (81.5 vs $50.0 \%) .{ }^{19}$ Abdominal pain was the most frequently reported TEAE (46.3\% with any naldemedine dose and $16.7 \%$ with PBO), with severe TEAEs occurring in one patient who received $1.0 \mathrm{mg}$ naldemedine (opioid withdrawal symptoms) and six patients who received $3.0 \mathrm{mg}$ naldemedine (abdominal pain, diarrhea, nausea/vomiting, and chills). A significant increase in spontaneous bowel movements (SBMs) within 24 hours of the initial dose was identified in patients receiving $0.3,1.0$, and $3.0 \mathrm{mg}$ doses of naldemedine compared to PBO. ${ }^{19}$ From these data, it was determined that the dose offering the best risk-benefit profile was $0.3 \mathrm{mg}$.

Subsequently, a Phase IIb (NCT: 01443403) randomized, double-blind, PBO-controlled parallel-group trial evaluated the efficacy and safety of once-daily dosing of $0.1,0.2$, and $0.4 \mathrm{mg}$ naldemedine vs PBO. ${ }^{15} \mathrm{~A}$ total of 244 patients with
OIC on a stable laxative regimen were randomized in a 1:1:1:1 fashion. Patients were given 28 days of treatment and then followed for an additional 28 days. The primary endpoint was the mean change in weekly SBM frequency compared to PBO. TEAEs, treatment-related AEs, and serious AEs (SAEs) were also compared between groups. ${ }^{15}$ Statistically significant increases in mean weekly SBMs from baseline to the last 2 weeks of treatment were identified in the 0.2 and $0.4 \mathrm{mg}$ naldemedine groups compared to PBO (least square mean increase 3.37 \pm 0.43 [standard error] SBMs and 3.64 \pm 0.44 SBMs vs $1.42 \pm 0.42, P=0.0014$ and $P=0.0003$, respectively) ${ }^{15}$ Seventy-one and two tenths percent of naldemedine $0.2 \mathrm{mg}$ patients and $66.7 \%$ of naldemedine $0.4 \mathrm{mg}$ patients, compared to $39.3 \%$ of $\mathrm{PBO}$ patients $(P=0.0005$ and $P=0.003$, respectively), were classified as SBM responders, defined as having $\geq 3 \mathrm{SBMs} /$ week and an increase of $\geq 1$ SBMs/ week from baseline. TEAEs were similar across treatment groups and numerically increased with naldemedine dose. The most common AEs were GI disturbances, including abdominal pain, diarrhea, flatulence, and nausea. ${ }^{15}$ There was one episode of chest pain, classified as a SAE in a patient receiving $0.1 \mathrm{mg}$ naldemedine. ${ }^{15}$ Based on these findings, naldemedine $0.2 \mathrm{mg}$ was selected as the optimal dose for Phase III analyses.

Two identical, multinational Phase III randomized, double-blind, PBO-controlled clinical trials (COMPOSE-I, NCT 01965158; COMPOSE-II, NCT 01993940) were conducted between 2013 and 2015 to assess the efficacy and safety of a daily dose of $0.2 \mathrm{mg}$ naldemedine. ${ }^{20}$ Adults on a stable opioid regimen who discontinued prior laxatives underwent stratified randomization based on their baseline opioid consumption. Patients received active treatment for 12 weeks, with subsequent monitoring for an additional 4 weeks. Based on an intention-to-treat population, COMPOSE-I ( $\mathrm{N}=273$ patients naldemedine; $272 \mathrm{PBO}$ ) and COMPOSE-II ( $\mathrm{N}=276$ patients naldemedine; $274 \mathrm{PBO})^{20}$ tested a durable response endpoint defined as $\geq 3$ SBMs/ week and an increase of $\geq 1$ SBMs/week from baseline for at least nine of the 12 treatment weeks, inclusive of at least three of the last 4 weeks. ${ }^{20}$ In both studies, there were significantly more responders in the naldemedine group compared to PBO (COMPOSE-I: 47.6 vs $34.6 \%, P=0.002$, and COMPOSE-II: 52.5 vs $33.6 \%, P<0.0001) .{ }^{20}$ There were also significant increases in the mean frequency of SBMs/ week from baseline to the last 2 weeks of treatment in favor of naldemedine (COMPOSE-I: least square mean increase $3.42 \pm 0.193$ [standard error] vs $2.12 \pm 0.192, P<0.0001$, and COMPOSE-II: $3.56 \pm 0.174$ vs $2.16 \pm 0.174, P<0.001) .{ }^{20}$ In 
both studies, there were similar numbers of TEAEs in both groups (COMPOSE-I: 49 vs 45\% and COMPOSE-II: 50 vs $48 \%$ ). A higher proportion of treatment-related AEs were noted in the naldemedine cohorts relative to $\mathrm{PBO}$ in both studies (COMPOSE-I: 22 vs 17\% and COMPOSE-II: 20 vs $11 \%) .{ }^{20}$ The most frequently reported AEs were diarrhea (COMPOSE-I: 7 vs 3\% and COMPOSE-II: 9 vs 2\%) and abdominal pain (COMPOSE-I: 6 vs $2 \%$ and COMPOSE-II: 5 vs 1\%). Two major cardiovascular AEs were documented, one in a treatment patient in COMPOSE-I and one in a PBO patient in COMPOSE-II. One patient receiving naldemedine in the COMPOSE-II study died from an opioid overdose leading to cardiopulmonary arrest. ${ }^{20}$ In addition, three patients in COMPOSE-I (two naldemedine and one PBO) experienced symptoms consistent with opioid withdrawal.

The final Phase III trial (COMPOSE-III, NCT 01965652) was a 52-week multicenter randomized, double-blind, PBOcontrolled study evaluating the long-term efficacy and safety of once daily $0.2 \mathrm{mg}$ naldemedine. ${ }^{21}$ The study population consisted of adults with OIC on stable doses of opioids, who may or may not have been on other laxatives. The primary outcome was safety and tolerability of naldemedine. Secondary endpoints assessed naldemedine efficacy. ${ }^{21}$ A total of 620 naldemedine and 620 PBO patients were included in the safety analysis. There were similar TEAEs in each group (68.4 vs $72.1 \%$, respectively). ${ }^{21}$ TEAEs that occurred in $>5 \%$ of patients and with a higher frequency in naldemedine than PBO groups included abdominal pain (8.2 vs 3.1\%), diarrhea (11.0 vs $5.3 \%)$, and vomiting (6.0 vs $3.1 \%) .{ }^{21}$ No associations between naldemedine and opioid withdrawal or decreased analgesia were evident. Patients in the treatment group had more frequent BMs/week, and this difference was statistically significant at all measured time points including at 52 weeks. $^{21}$

\section{Place in therapy}

Naldemedine is the third PAMORA FDA approved for OIC in adults with chronic noncancer pain. Based on the aforementioned clinical studies, it appears efficacious with a favorable safety profile. It can be used in patients with renal impairment, including those with end-stage renal disease, as well as in patients with mild or moderate hepatic impairment. ${ }^{14}$ Naldemedine can be taken with or without food and with or without additional laxatives. ${ }^{14}$ It can be taken with any opioid for chronic noncancer pain including methadone. ${ }^{14}$ Naldemedine should be avoided in patients with known or suspected GI obstruction or those at risk for recurrent obstruction. ${ }^{14}$

Currently, there are no head-to-head trials comparing naldemedine to the other FDA-approved PAMORAs. Treatment outcomes (Table 2) and side effect profiles (Table 3) of the FDA-approved orally dosed PAMORAs are similar. Therefore, initially, the choice of which PAMORA to use will depend on patient and prescriber preferences as well as cost and coverage.

\section{Conclusion}

OIC is common in adults on opioids, and the PAMORAs have emerged as a useful therapeutic class to counteract the constipating effects of opioids on the GI tract while preserving

Table 2 Treatment outcomes of FDA-approved orally dosed PAMORAs in patients with OIC and noncancer pain

\begin{tabular}{|c|c|c|c|}
\hline & Study primary endpoint & $\begin{array}{l}\text { Results active } \\
\text { comparator (\%) }\end{array}$ & $\begin{array}{l}\text { Results } \\
\text { placebo (\%) }\end{array}$ \\
\hline Methylnaltrexone 450 mg PO daily ${ }^{22}$ & $\geq 3 \mathrm{SBM} /$ week + increase $\geq 1$ SBM/week for $3 / 4$ weeks & 52 & 38 \\
\hline Naldemedine 0.2 mg PO daily ${ }^{20}$ & $\geq 3$ SBM/week + increase I SBM from baseline for & 47.6 & 34.6 \\
\hline COMPOSE I trial & $9 / 12$ weeks +3 of last 4 weeks & 52.5 & 33.6 \\
\hline \multicolumn{4}{|l|}{ COMPOSE II trial } \\
\hline Naloxegol 25 mg PO daily ${ }^{12}$ & $\geq 3 \mathrm{SBM} /$ week + increase I SBM from baseline for & 42 & 29 \\
\hline $\begin{array}{l}\text { Naloxegol } 12.5 \text { mg PO daily'2 } \\
\text { (KODIAC-04/05 aggregated) }\end{array}$ & $9 / 12$ weeks +3 of last 4 weeks & 38 & 29 \\
\hline
\end{tabular}

Abbreviations: FDA, US Food and Drug Administration; OIC, opioid-induced constipation; PAMORAs, peripherally acting $\mu$-opioid receptor antagonists; SBM, spontaneous bowel movement.

Table 3 Side effect profile of FDA-approved orally dosed PAMORAs in patients with OIC and noncancer pain at up to 12 weeks of therapy

\begin{tabular}{llll}
\hline & Abdominal pain (placebo, \%) & Diarrhea (placebo, \%) & Vomiting (placebo, \%) \\
\hline Methylnaltrexone 450 mg PO daily & $14(10)$ & $5(2)$ & $3(2)$ \\
Naldemedine 0.2 mg PO daily'14 & II (5) & $7(3)$ & $3(2)$ \\
Naloxegol 25 mg PO daily'3 & $21(7)$ & $9(5)$ & $8(5)$ \\
Naloxegol I2.5 mg PO daily & $12(7)$ & $8(5)$ & $7(5)$ \\
\hline
\end{tabular}

Abbreviations: FDA, US Food and Drug Administration; OIC, opioid-induced constipation; PAMORAs, peripherally acting $\mu$-opioid receptor antagonists. 
analgesia. Naldemedine is the third PAMORA to be FDA approved for the treatment of OIC in adults with chronic noncancer pain. Based on multiple well-designed Phase II and Phase III clinical trials, naldemedine appears to be a safe and effective option for OIC patients who have failed a trial of over the counter laxatives. Future research is needed to compare naldemedine to the other available PAMORAs.

\section{Disclosure}

DMB serves as a consultant/advisor and/or is on the speakers' bureau for Allergan, AstraZeneca, Daiichi Sankyo, Shionogi, Medscape, GI Health Foundation, Ironwood, Salix, and Synergy Pharmaceuticals. EKS reports no conflicts of interest in this work.

\section{References}

1. Nahin RL. Estimates of pain prevalence and severity in adults: United States, 2012. J Pain. 2015;16(8):769-780.

2. Boudreau D, Von Korff M, Rutter CM, et al. Trends in long-term opioid therapy for chronic non-cancer pain. Pharmacoepidemiol Drug Saf. 2009;18(12):1166-1175.

3. Brenner DM, Stern E, Cash BD. Opioid-related constipation in patients with non-cancer pain syndromes: a review of evidence-based therapies and justification for a change in nomenclature. Curr Gastroenterol Rep. 2017;19(3):12.

4. Camilleri M, Drossman DA, Becker G, Webster LR, Davies AN, Mawe GM. Emerging treatments in neurogastroenterology: a multidisciplinary working group consensus statement on opioid-induced constipation. Neurogastroenterol Motil. 2014;26(10):1386-1395.

5. Lacy BE, Mearin F, Chang L, et al. Bowel disorders. Gastroenterology. 2016;150(6):1393-1407.e1395.

6. Kalso E, Edwards JE, Moore RA, McQuay HJ. Opioids in chronic noncancer pain: systematic review of efficacy and safety. Pain. 2004;112(3): 372-380.

7. Cook SF, Lanza L, Zhou X, et al. Gastrointestinal side effects in chronic opioid users: results from a population-based survey. Aliment Pharmacol Ther. 2008;27(12):1224-1232.

8. Leppert W. Emerging therapies for patients with symptoms of opioidinduced bowel dysfunction. Drug Des Devel Ther. 2015;9:2215-2231.

9. Bell TJ, Panchal SJ, Miaskowski C, Bolge SC, Milanova T, Williamson R. The prevalence, severity, and impact of opioid-induced bowel dysfunction: results of a US and European Patient Survey (PROBE 1). Pain Med. 2009;10(1):35-42.
10. Amitiza ${ }^{\circledR}$ (lubiprostone) [prescribing information]. Bethesda, MD: Sucampo Pharmaceuticals, Inc; 2013.

11. Camilleri M, Bharucha AE, Ueno R, et al. Effect of a selective chloride channel activator, lubiprostone, on gastrointestinal transit, gastric sensory, and motor functions in healthy volunteers. Am J Physiol Gastrointest Liver Physiol. 2006;290(5):G942-G947.

12. Chey WD, Webster L, Sostek M, Lappalainen J, Barker PN, Tack J. Naloxegol for opioid-induced constipation in patients with noncancer pain. N Engl J Med. 2014;370(25):2387-2396.

13. Movantik $\mathbb{B}$ (naloxegol) [package insert]. Wilmington, DE: AstraZeneca Pharmaceuticals LP; 2014.

14. SYMPROIC $®$ (naldemedine) [package insert]. Florham Park, NJ: Shionogi Inc; 2017.

15. Webster LR, Yamada T, Arjona Ferreira JC. A phase 2b, randomized, double-blind placebo-controlled study to evaluate the efficacy and safety of naldemedine for the treatment of opioid-induced constipation in patients with chronic noncancer pain. Pain Med. 2017;18(12):2350-2360.

16. Federal Drug Administration. Symproic (Naldemedine Tosylate) Medical Report. Silver Spring, MD: Federal Drug Administration; 2017.

17. Markham A. Naldemedine: first global approval. Drugs. 2017;77(8): 923-927.

18. Kanemasa T, Ono H, Nakamura A, Morioka Y, Hasegawa M. Effects of naldemedine: a peripherally acting mu-opioid receptor antagonist in rat models of opioid-induced constipation [abstract no. 1322]. Am J Gastroenterol. 2015;110(suppl 1):S81.

19. Webster L, Nagata T, Yamada T, Arjona Ferreira J. (479) A phase 2a, randomized, double-blind, placebo-controlled, single ascending-dose study to evaluate the safety and efficacy of naldemedine in patients with chronic non-cancer pain and opioid-induced bowel dysfunction. J Pain. 2016;17(4S):S94.

20. Hale M, Wild J, Reddy J, Yamada T, Arjona Ferreira JC. Naldemedine versus placebo for opioid-induced constipation (COMPOSE-1 and COMPOSE-2): two multicentre, phase 3, double-blind, randomised, parallel-group trials. Lancet Gastroenterol Hepatol. 2017;2(8): $555-564$.

21. Webster L, Nalamachu S, Yamada T, et al. Long-term safety and efficacy of naldemedine for the treatment of opioid-induced constipation in subjects with chronic non-cancer pain receiving opioid therapy: results from a 52-week phase 3 clinical trial [abstract no. 7]. Postgrad Med. 2016;128(suppl 2):5.

22. Rauck R, Slatkin NE, Stambler N, Harper JR, Israel RJ. Randomized, double-blind trial of oral methylnaltrexone for the treatment of opioidinduced constipation in patients with chronic noncancer pain. Pain Pract. 2017;17(6):820-828.

23. RELISTOR $®$ (methylnaltrexone bromide) [package insert]. Bridgewater, NJ: Salix Pharmaceuticals, a division of Valeant Pharmaceuticals North America LLC; 2016.
Journal of Pain Research

\section{Publish your work in this journal}

The Journal of Pain Research is an international, peer reviewed, open access, online journal that welcomes laboratory and clinical findings in the fields of pain research and the prevention and management of pain. Original research, reviews, symposium reports, hypothesis formation and commentaries are all considered for publication

\section{Dovepress}

The manuscript management system is completely online and includes a very quick and fair peer-review system, which is all easy to use. Visit http://www.dovepress.com/testimonials.php to read real quotes from published authors. 